\title{
The Geometry of a Covariant Expansion in Chiral Theories with Nucleons
}

\author{
Michael Daniel \\ Department of Physics, Division of Theoretical Physics, Panepistimiopolis, Athens 621, Greece
}

\begin{abstract}
We deal with the geometrical foundations of a covariant scheme, which is developed to give a "co-ordinate independent" perturbation expansion of $S U(2) \times S U(2)$ chiral theories with pions and nucleons. The pion fields play the role of local co-ordinates on a 3-D manifold with constant curvature in isospace. The presence of the nucleon isospinor field forces us to deal with the problem of endowing the manifold with a spin structure. In this way the nucleon isospinor is accommodated in the fiber space of the principal fiber bundle of the tangent bundle of the manifold.
\end{abstract}

\section{Introduction}

The non-linear realizations of the chiral group $G=S U(2) \times S U(2)$ are studied from the geometric point of view in [1]. In this reference the 3-dimensional non-linear realization associated with the pion isovector field is considered as a group of co-ordinate transformations on a 3-dimensional isospace of constant curvature leaving invariant the line element. In this treatment the pion field components are taken to be the co-ordinates in the curved isospace. The various non-linear models (i.e. chiral invariant Lagrangian densities which are functions of the pion field only) result from a specific choice of the co-ordinate system used to parametrize the manifold. It is known, however, that the $S$-matrix elements should be independent of the choice of the pion field in term of which the Lagrangian density is defined. A co-ordinate independent formulation of perturbation theory is developed in $[2,3]$. In the present article we would like to extend the formalism of $[2,3]$ to cover the case of $S U(2) \times S U(2)$ invariant Lagrangian densities which are functions of the pion isovector as well as the nucleon isospinor fields. Consequently we are forced to deal with the problem of "laying down a spinor field" upon the manifold under consideration, that is, it is necessary to endow the manifold with a spin structure. This structure is defined by means of a field of driads. The dreibein fields, therefore, play an essential role in our formalism. 
In conclusion we remark that the present work provides the necessary geometric background for the work of Reference [4].

\section{The Spin Structure}

Consider the 3 -sphere $S^{3} \subset R^{4}$ of radius $F_{\pi}, F_{\pi}$ being the pion decay constant. The group $G$ acts transitively on $S^{3}$. As a manifold $S^{3}$ is orientable. Thus, the tangent bundle of $S^{3}$ has the rotation group $S O(3)$ as structural group. The tangent bundle is also trivial [5]. There exist three cross-sections of the bundle which are no-where linearly dependent [6]. Applying the Gram-Schmidt process we obtain an orthonormal set of cross-sections $s_{1}, s_{2}, s_{3}$ i.e. $s_{a}(\pi) \cdot s_{b}(\pi)=\delta_{a b}$ for each $\pi=\left(\pi^{1}, \pi^{2}, \pi^{3}\right) \in S^{3}$. We recall that the pion fields are taken to be the co-ordinates on $S^{3}$. The inner product is defined in terms of the Riemannian metric $g=g_{i j} d \pi^{i} \otimes d \pi^{j}$ of the manifold. Now the cross-sections of the tangent bundle are vector fields. Hence, with respect to a local chart the cross-sections acquire the form

$$
s_{a}(\pi)=s_{a}^{i}(\pi) \frac{\partial}{\partial \pi^{i}}
$$

so that

$$
s_{a}(\pi) \cdot s_{b}(\pi)=g\left(\frac{\partial}{\partial \pi^{i}}, \frac{\partial}{\partial \pi^{j}}\right) s_{a}^{i}(\pi) s_{b}^{j}(\pi)=g_{i j}(\pi) s_{a}^{i}(\pi) s_{b}^{j}(\pi) .
$$

Thus, the dreibein fields of [3] are the components of the cross-sections $s_{a}$ with respect to a local chart.

Let $\xi$ be the principal fibre bundle of the tangent bundle with structural group $S O(3)$. The total space of $\xi$ is denoted by $E(\xi)$ and the base $B$ is the manifold itself. If it is possible to replace the structural group $S O(3)$ by its 2-fold covering group $\operatorname{Spin}(3)$, then one says that $S^{3}$ can be given a spin structure. It can be shown that an orientable compact Riemannian manifold $M$ admits a spin structure if and only if its second Whitney characteristic class $C_{2}(M)$ is null $[7,8]$. For the 3-sphere $C_{2}\left(S^{3}\right)=0$ holds [5], and the manifold is parallelizable. Thus, $S^{3}$ can be given a spin structure. This implies the existence of a pair $(\eta, f)$ consisting of (i) a principal bundle $\eta$ over $B$ with $\operatorname{Spin}(3)$ as a structural group, and (ii) a map $f: E(\eta) \rightarrow E(\xi)$ such that the following diagram is commutative [8].

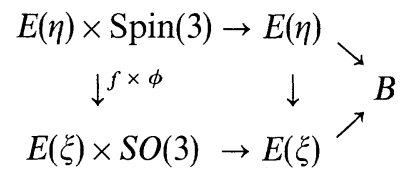

where $\phi$ is the standard $\operatorname{Spin}(3) \rightarrow S O(3)$ homomorphism. Consequently with regards to the bundle $\eta$ the fiber above each point $P \in B$ is a 2-dimensional complex space (spin space) with $\operatorname{Spin}(3)$ structure. This fact will enable us to deal with the covariant perturbation expansion of chiral theories with pions and nucleons. It is clear that the spin structure of the manifold is indispensable in order to accommodate the nucleon isospinor field. 


\section{Geometry of the Bundle}

We would like to examine the spin space in some detail. We introduce the Clifford algebra $\mathscr{T}$ (over the field $R$ ), which contains the unit element $e_{0}$ and is generated by $e_{0}$ and three other elements $e_{1}, e_{2}, e_{3}$. The elements $\left\{e_{a}\right\}$ is a system of $2 \times 2$ matrices

$$
e_{a}=\left(e_{a B}^{A}\right)
$$

(the indices $a, b, \ldots$, and $A, B, \ldots$ are tensor and spinor indices respectively). The elements $e_{0}, e_{1}, e_{2}, e_{3}$ satisfy

$$
e_{0} e_{0}=e_{0}, \quad e_{0} e_{a}=e_{a} e_{0}=e_{a}, \quad e_{a} e_{b}+e_{b} e_{a}=0 \quad(a \neq b),
$$

and

$$
e_{a}^{2}=e_{0} \quad(a, b=1,2,3) .
$$

The regular elements of $\mathscr{T}$ form a multiplicative group denoted by $\mathscr{T}^{*}$. Now, if $x \in \mathscr{T}^{*}$, the mapping $\mathscr{T} \ni y \rightarrow x y x^{-1}$ is an endomorphism of $\mathscr{T}$, which may be represented by a matrix $\Theta(x)$. Moreover the map $x \rightarrow \Theta(x)$ is a representation of $\mathscr{T}^{*}$. Let $\mathscr{M}$ be a vector subspace of $\mathscr{T}$ which is spanned by $e_{1}, e_{2}, e_{3}$. Consider the set of those elements $x \in \mathscr{T}^{*}$ which are such that $\Theta(x)$ maps $\mathscr{M}$ into itself. This is a subgroup of $\mathscr{T}^{*}$. We are, now, in position to define the Spin (3) group.

$\operatorname{Spin}(3)$. Let $H$ be the group of elements $x \in \mathscr{T}^{*}$ such that $\Theta(x)(\mathscr{M}) \subset \cdot \mathscr{M}$ with $\operatorname{det} \Theta(x)=1$. The component of $e_{0}$ in $H$ is called the spinor group, Spin(3).

The contraction of $\Theta(x)$ to $\mathscr{M}$ is an endomorphism of $\mathscr{M}$ denoted by $\phi(x)$. $\phi(x)$ is represented by an orthogonal matrix in terms of the basis $\left\{e_{1}, e_{2}, e_{3}\right\}$ i.e. $\phi: \operatorname{Spin}(3) \rightarrow S O(3)$

$$
\phi(x) e_{a}=R_{a b} e_{b} \text {, with } R R^{T}=1 .
$$

Thus, for each $Q \in \operatorname{Spin}(3) R \in S O(3)$ such that

$$
Q e_{a} Q^{-1}=R_{b a} e_{b}
$$

The Pauli matrices $\tau_{a}$ form a natural basis for $\mathscr{M}$. With respect to this basis let $\Gamma^{i}(\pi)=s_{a}^{i}(\pi) \tau_{a}$. Then

$$
\left\{\Gamma^{i}(\pi), \Gamma^{i}(\pi)\right\}_{+}=2 g^{i j}(\pi) e_{0}
$$

which describes the Clifford algebra on the curved isospace under consideration.

With the field of cross-sections $s_{a}$ is associated a spinor basis $h$ which is normalized to unity. A contravariant spinor $\Psi$ is defined as follows:

$$
\Psi\left(h Q^{-1}\right)=Q \Psi(h) \quad Q \in \operatorname{Spin}(3)
$$

where $\Psi(h)=\left(\Psi^{A}\right)$. In terms of the components $\Psi^{A}$ the above transformation equation reads

$$
\Psi^{\prime A}=Q_{B}^{A} \Psi^{B} .
$$

The contravariant spinors form a complex vector space $S$. Similarly one can introduce the dual space $S^{*}$, the space of covariant spinors. A covariant spinor 
is defined as follows:

$$
\Phi\left(h Q^{-1}\right)=\Phi(h) Q^{-1} \quad Q \in \operatorname{Spin}(3)
$$

where $\Phi(h)=\left(\Phi_{A}\right)$. The components $\Phi_{A}$ obey the transformation law

$$
\Phi_{A}^{\prime}=\Phi_{B}\left(Q^{-1}\right)_{A}^{B} .
$$

The inner product is defined as: $\Phi \cdot \Psi=\Phi_{A} \Psi^{A}$.

For the basis $h$ the following relations hold

$$
h_{A} \cdot h_{B}=\varepsilon_{A B}, \quad h^{A} \cdot h^{B}=\varepsilon^{A B}, \quad h^{A} \cdot h_{B}=\delta_{B}^{A}
$$

where

$$
\varepsilon_{A B}=\varepsilon^{A B}=\left(\begin{array}{cc}
0 & 1 \\
-1 & 0
\end{array}\right) .
$$

Connections. A connection on $\left.\xi\right|_{U}$ (where $\{U\}$ is a collection of open sets which covers $S^{3}$ ) is defined by

$$
\left(\nabla_{U}\right)_{X}\left(s_{a}\right)=C_{a b}(X) s_{b}
$$

where $X$ is a vector field on $U$. With respect to a local co-ordinate chart $\{\pi\}$ on $U$ we obtain, using for convenience $\nabla_{k}$ instead of $\left(\nabla_{U}\right)_{X}$,

$$
\nabla_{k}\left(s_{a}\right)=\nabla_{k}\left(s_{a}^{i} \partial / \partial \pi^{i}\right)=\left(s_{a, k}^{i}+\Gamma_{m k}^{i} s_{a}^{m}\right) \partial / \partial \pi^{i}
$$

where $\Gamma_{j k}^{i}$ are the Christoffel symbols associated with the metric $g_{i j}$. Hence the components of the dreibein fields satisfy the differential equation

$$
s_{a, k}^{i}=C_{a b k} s_{b}^{i} .
$$

On the other hand the connection $\nabla$ must be compatible with the metric. Hence

$$
0=d\left(s_{a} \cdot s_{b}\right)=\left(\nabla s_{a} \cdot s_{b}\right)+\left(s_{a} \cdot \nabla s_{b}\right)=C_{a b}+C_{b a} .
$$

It follows that the connection metric $\left(C_{a b}\right)$ is skew-symmetric i.e. $C_{a b}=C_{[a b]}$.

A spinorial connection is an infinitesimal connection in the fiber space of $\eta$. To the dreibein field connection $C$ there corresponds a spinorial connection $\Omega$. $C$ is a 1 -form in the fiber space of $\xi$ which takes values in the Lie algebra of $S O(3)$, whereas $\Omega$ is a 1 -form in the fiber space of $\eta$, which takes values in the Lie algebra of $\operatorname{Spin}(3)$. Hence

$$
\Omega=\phi^{-1} C \text {. }
$$

Using the matrix representation of $C$ and making use of the standard homomorphism between $\operatorname{Spin}(3)$ and $S O(3)$ as obtained from (1) we deduce that

$$
\Omega=(1 / 8) C_{a b}\left[\tau_{a}, \tau_{b}\right] \text {. }
$$

So far we have developed some of the local geometry of the manifold. It is necessary, however, to find consistency conditions which make the global definition of the connection possible. To this end we consider systems $\left(U,\left\{s_{a}\right\}, C\right)$, and $\left(U^{\prime},\left\{s_{a}^{\prime}\right\}, C^{\prime}\right)$ with $U \cap U^{\prime} \neq \emptyset$. Then for any vector field $Y$ defined on the intersection 
$U \cap U^{\prime}$ the computation of $\nabla Y$ in either connection must yield the same results. On the overlap we have

$$
s_{a}^{\prime}(\pi)=R_{a b}(\pi) s_{b}(\pi), \quad \pi \in U \cap U^{\prime}
$$

Thus

$$
\nabla s_{a}^{\prime}=(d R+R C)_{a b}\left(R^{-1} s^{\prime}\right)_{b}=C_{a b}^{\prime} s_{b}^{\prime} .
$$

This yields the transformation law for $C$, namely

$$
C^{\prime}=(d R+R C) R^{-1} \text {. }
$$

Now, with regards to the spin basis we have: $\nabla_{X} h_{A}=-\Omega_{A}^{B} h_{B}$. Dreibein field rotations induce $S p i n$ (3) transformations in the spin space. Thus, for every $R \in S O(3)$, there exists $Q \in \operatorname{Spin}(3)$ such that the local basis transforms as $h \rightarrow h Q^{-1}$. The connection $\Omega$ transforms like

$$
\Omega \rightarrow \Omega^{\prime}=Q \Omega Q^{-1}-Q\left(d Q^{-1}\right) .
$$

Let $\Psi$ be a contravariant spinor. Then $\nabla_{k} \Psi=\Psi_{{ }_{k}}^{A} h_{A}$, where $\Psi_{\cdot k}^{A}=\Psi_{{ }_{k}}^{A}-\Omega_{B k}^{A} \Psi^{B}$. Similarly for a covariant spinor $\Phi$ the following holds: $\nabla_{k} \Phi=\Phi_{A \cdot k} h^{A}$ where $\Phi_{A \cdot k}=\Phi_{A, k}+\Phi_{B} \Omega_{A k}^{B}$. Here we have made use of the fact that $\left(\nabla_{k} h^{B}\right) \cdot h_{A}=-h^{B} \cdot\left(\nabla_{k} h_{A}\right)$. It is clear that due to the transformation properties of the connection $\Omega, \Psi_{{ }_{k}}^{A}$ and $\Phi_{A \cdot k}$ transform like $\psi^{A}$ and $\Phi_{A}$ respectively.

Parallel Transfer. Let $\gamma$ be a congruence of geodesics on $S^{3}$. The family of tangent vectors $\dot{\gamma}(t)$ is parallel with respect to $\gamma$ by definition. Hence $\nabla_{X}(X)_{\gamma(t)}=0$, where $X=\dot{\gamma}(t)$. In terms of a local chart $\left\{\pi^{i}\right\}$ the vector $X$ acquires the form $X=\dot{\zeta}^{k} \partial / \partial \pi^{k}$ and the previous equation reduces to

$$
\ddot{\zeta}^{k}+\Gamma_{i j}^{k} \dot{\zeta}^{i} \dot{\zeta}^{k}=0
$$

which is the familiar equation for the geodesics used in [2].

We turn, now, to the condition for the parallel transfer of the spin space. A family of contravariant spinor fields $\Psi(t)$ is said to be parallel with respect to the same congruence of geodesics if

$$
\nabla_{X}(\Phi)_{\gamma(t)}=0 \text {. }
$$

Writing $\Psi=\Psi^{A} h_{A}$ we readily obtain

$$
d \Psi^{A} / d t-\Omega_{B k}^{A} \Psi^{B} d \zeta^{k} / d t=0 .
$$

Similarly for a covariant spinor field $\Phi(t)$ propagated parallely along the geodesics we have

$$
\nabla_{X}(\Phi)_{\gamma(t)}=0
$$

which yields

$$
d \Phi_{A} / d t+\Phi_{B} \Omega_{A k}^{B} d \zeta^{k} / d t=0 .
$$

Remark. Equations (5)-(7) constitute a complete set of relations to be used in a covariant expansion of any quantity which is a co-ordinate scalar. 


\section{The Model}

Consider a Lorentz invariant Lagrangian density with $S U(2) \times S U(2)$ symmetry, which is a function of the pion isovector field as well as the nucleon isospinor. The most general expression for such a family of Lagrangian densities with minimal coupling (i.e. with at most two derivatives) is given by

$$
\begin{aligned}
\mathscr{L}_{\kappa}= & \frac{1}{2} g\left(\partial / \partial \pi^{i}, \partial / \partial \pi^{j}\right) \partial_{\mu} \pi^{i} \partial^{u} \pi^{j} \\
& +i \bar{\Phi} \cdot \not D \Psi-m \bar{\Phi} \cdot \Psi+\kappa / 2 \bar{\Phi} \cdot \gamma_{5} I \cdot \Psi, \quad\left(\bar{\Phi}=\Phi \otimes \gamma^{0}\right)
\end{aligned}
$$

where $\not D=\partial \pi^{i} \nabla_{i}$, and $I^{\prime}=\partial \pi^{i} \Gamma_{i}$. Here the covariant spinor $\Phi$ is identified with the conjugate complex to the contravariant spinor $\Psi$. Under co-ordinate transformations $\pi \rightarrow \pi^{\prime}(\pi)$ the quantities $\Gamma^{i}$ transform like the components of a contravariant vector, that is

$$
\Gamma^{\prime i}\left(\pi^{\prime}\right)=\left(\partial \pi^{i} / \partial \pi^{j}\right) \Gamma^{j}(\pi) .
$$

However, because the group $G$ induces isometric transformations on $S^{3}$, we deduce that there exists a matrix $Q \in \operatorname{Spin}(3)$ such that

$$
\Gamma^{i}\left(\pi^{\prime}\right)=Q^{-1} \Gamma^{i}(\pi) Q .
$$

Hence bilinear quantities, in terms of the spinor fields, which are constructed with the $\Gamma^{i}$ transform as tensors [1]. It is, therefore, clear that $\mathscr{L}_{\kappa}$ is a co-ordinate scalar.

Consider the total action $\mathfrak{I}[\pi, \Psi, \Phi]=\int \mathscr{L}_{\kappa}(x) d^{4} x$, with $\kappa=F_{\pi}^{-1}{ }^{1}$ Our aim is to construct a covariant perturbation expansion of $\mathfrak{I}$ with the terms of the expansion transforming covariantly under pion field redefinition (recall that a pion field redefinition is tantamount to co-ordinate transformations on $S^{3}$ ). To this end a classical pion field $\vec{\phi}$ is introduced, which satisfies the differential equation

$$
\frac{\delta \mathfrak{I}_{1}[\pi]}{\delta \pi^{i}(x)}=-J_{i}(x)
$$

where $\vec{J}(x)$ is a classical source associated with the field $\vec{\phi}$, and $\mathfrak{I}=\mathfrak{I}_{1}+\mathfrak{I}_{2}$ where $\mathfrak{I}_{1}$ corresponds to pure pion self-interaction. This terminology is qualified by the fact that the classical field $\vec{\phi}$ satisfies

$$
\phi^{i}(x)=\left[\frac{\left\langle 0^{+}\left|\pi^{i}(x)\right| 0^{-}\right\rangle}{\left\langle 0^{+} \mid 0^{-}\right\rangle}\right]_{J} .
$$

In the following we intend to give a covariant expansion of $\mathfrak{I}[\pi, \psi, \Phi]$ around $\vec{\phi}$. This is achieved in two steps:

(i) First the functional $\mathfrak{T}[\pi, \psi, \Phi]$ is expanded around $\vec{\phi}$ yielding

$$
\begin{aligned}
\mathfrak{I}[\pi, \Psi, \Phi]= & \mathfrak{T}_{1}[\phi]+\sum_{n=1}^{\infty} \frac{1}{n !} \mathfrak{T}_{1,\{n\}}[\phi] \chi^{\{n\}} \\
& +\bar{\Phi} \cdot \mathfrak{A} \cdot \Psi+\sum_{n=1}^{\infty} \frac{1}{n !} \bar{\Phi}_{A}\left(\mathfrak{H}_{,\{n\}}[\phi]\right)_{B}^{A} \Psi^{B} \chi^{\{n\}}
\end{aligned}
$$

1 This choice for $\kappa$ implies the absence of pion-nucleon interactions in the case of vanishing curvature 
where $\pi^{i}=\phi^{i}+\chi^{i},\{n\}$ is a shorthand for the set of indices $k_{1} \ldots k_{n}$, and $\chi^{\{n\}}=\chi^{k_{1}} \ldots \chi^{k_{n}}$. Moreover $\mathfrak{A}$ is a spinorial tensor of valence $\left(\begin{array}{l}1 \\ 1\end{array}\right)$, the components of which are given by

$$
\mathfrak{N}_{B}^{A}\left(x, x^{\prime}\right)=\frac{\vec{\delta}}{\delta \bar{\Phi}_{A}(x)} \mathfrak{T}_{2}[\pi, \Psi, \Phi] \frac{\overleftarrow{\delta}}{\delta \Psi^{B}\left(x^{\prime}\right)}
$$

with the arrows indicating left and right functional derivatives which take into account the anticommuting nature of the spinor fields. We remark that in general summation over repeated indices implies integration over their associated spacetime co-ordinates.

(ii) We now turn into the integral curves of (4)-(6) .Any integral curve of (4) is determined by a point on the manifold together with a direction at that point. The former is taken to be an arbitrary point $P\left(\phi^{i}\right) \in S^{3}$ with $\left.\zeta^{i}(t)\right|_{t=0}=\phi^{i}$, and the direction is determined by a unit tangent vector at $P$, namely $\left.\frac{d \zeta^{i}}{d t}\right|_{t=0}=\frac{\Gamma^{i}}{\mathrm{~s}}$, where $\Gamma_{\cdot i}$ is obtained by differentiating the biscalar quantity

$$
\Gamma(\pi, \phi)=s \int_{0}^{s} d t \frac{1}{2} g_{i j}[\zeta(t)] \frac{d \zeta^{i}}{d t} \frac{d \zeta^{j}}{d t} .
$$

Thus, we obtain

$$
\zeta^{i}(t)=\phi^{i}+\frac{\Gamma^{i}}{s}+\left.\frac{1}{2 !} \frac{d^{2} \zeta^{i}}{d t^{2}}\right|_{t=0} t^{2}+\ldots
$$

The coefficients of $t^{n}(n>1)$ are given from (4) by differentiation with respect to $t$ and replacing the second and higher derivatives of $\zeta^{i}$ by means of (4) and the resulting equations. Putting $t=s$ we finally obtain:

$$
\pi^{i}=\phi^{i}-\sum_{n=1}^{\infty} n !^{-1} \Gamma_{\{n\}}^{i} \Gamma^{\{n\}}
$$

where $\Gamma_{\{n\}}^{i} \equiv \Gamma_{k_{1} \ldots k_{n}}^{i}$ are the generalised Christoffel symbols with $\Gamma_{k}^{i}=-\delta_{k}^{i}$ and $\left.\zeta^{i}(t)\right|_{t=s}=\pi^{i}$.

Similarly the integral curves of the first order equations (5) and (6) are determined by a "point", which is taken to be the corresponding point in the fiber space above $P$. Applying the same procedure as above we obtain:

$$
\begin{aligned}
& \Psi^{A}=\psi^{A}+\sum_{n=1}^{\infty} n !^{-1} \Omega_{B\{n\}}^{A} \psi^{B} \Gamma^{\{n\}} \\
& \Phi^{A}=\omega_{A}+\sum_{n=1}^{\infty} n !^{-1} \omega_{B} \bar{\Omega}_{A\{n\}}^{B} \Gamma^{\{n\}}
\end{aligned}
$$

where $\Omega_{B\{n\}}^{A}$ and $\bar{\Omega}_{B\{n\}}^{A}$ are the generalised connections in spin space:

$$
\begin{aligned}
\bar{\Omega}_{B k}^{A} & =-\Omega_{B k}^{A} \\
\Omega_{B k l}^{A} & =\frac{1}{2} \sigma\left[\Omega_{B k, l}^{A}+\Omega_{c k}^{A} \Omega_{B l}^{c}\right]-\Omega_{B m}^{A} \Gamma_{k l}^{m} \\
\bar{\Omega}_{B k l}^{A} & =\frac{1}{2} \sigma\left[-\Omega_{B k, l}^{A}+\Omega_{c k}^{A} \Omega_{B l}^{C}\right]+\Omega_{B m}^{A} \Gamma_{k l}^{m}
\end{aligned}
$$


where $\sigma$ before an expression indicates symmetrization with respect to the chiral indices $k, l, m, \ldots$. Inserting (10), (11), and (12) in (9) one readily obtains the covariant expansion:

$$
\begin{aligned}
\mathfrak{T}[\pi, \Psi, \Phi]= & \mathfrak{I}_{1}[\varphi]+\sum_{n=1}^{\infty} n !^{-1} \mathfrak{T}_{1 ; k_{1} k_{2} \ldots k_{n}}[\varphi] \Gamma^{k_{1}} \ldots \Gamma^{k_{n}}+\bar{\omega} \cdot \mathfrak{U}[\varphi] \cdot \psi \\
& +\sum_{n=1}^{\infty} n !^{-1} \bar{\omega} \cdot \mathfrak{U}_{k_{k_{1}} \cdots k_{n}}[\varphi] \cdot \psi \Gamma^{k_{1}} \ldots \Gamma^{k n}
\end{aligned}
$$

where

$$
\begin{aligned}
& \mathfrak{U}_{B . k}^{A}=\mathfrak{U}_{B, k}^{A}+\mathfrak{U}_{C}^{A} \Omega_{B k}^{C}-\Omega_{C k}^{A} \mathfrak{U}_{B}^{C} \\
& \mathfrak{U}_{B . k_{1} k_{2}}^{A}=\left(\mathfrak{U}_{B . k_{1}}^{A}\right)_{, k_{2}}+\mathfrak{U}_{C . k_{1}}^{A} \Omega_{B k_{2}}^{C} \mathfrak{U}_{B . k_{1}}^{C}-\Gamma_{k_{1} k_{2}}^{m} \mathfrak{U}_{B . m}^{A}
\end{aligned}
$$

and so on.

\section{Covariant Perturbation Theory ${ }^{2}$}

We shall develop, now, a covariant perturbation theory using functional integral techniques. The starting point is the generating functional for connected Green's functions, $\amalg[J, \eta]$, given by

$$
\begin{aligned}
\exp i \omega[J, \eta]= & N^{-1} \int \prod_{x} \prod_{i} d \pi^{i} \sqrt{g(\pi)} \prod_{A} d \Psi^{A} \prod_{B} d \Phi_{B} \\
& \cdot \exp i\left\{\mathfrak{I}[\pi, \Psi, \Phi]+\int[\vec{J} \cdot \vec{\pi}+\bar{\Phi} \cdot \eta+\bar{\eta} \cdot \Psi] d x\right\}
\end{aligned}
$$

where $\eta$ is a spinor source of the anticommuting type, and $N$ is a normalization factor fixed by the condition $\exp i \amalg[0]=1$. We remark that the factor $\sqrt{g(\pi)}$ with $g(\pi)=\operatorname{det}\left(g_{i j}(\pi)\right)$ is required to maintain a formal invariance of the functional measure with respect to the pion field redefinitions. The covariant expansion for $\mathfrak{I}$ as well as the expansions (10), (11), and (12) are, now, inserted. After changing the integration variables from $\pi^{i}$ to $\Gamma^{i}$. and from $\left(\Phi_{A}, \Psi^{A}\right)$ to $\left(\omega_{A}, \psi^{A}\right)$ all the exponentials are expanded out except for the term that involves

$$
\frac{1}{2} \mathfrak{I}_{1 ; k_{1} k_{2}}[\phi] \Gamma^{k_{1}} \Gamma^{k_{2}}+\bar{\omega} \cdot \mathfrak{U} \cdot \psi+\bar{\omega} \cdot \eta+\bar{\eta} \cdot \psi .
$$

In this way the functional integral is reduced to a series of functional integrals that can be calculated (formally) in a standard way. These integrals are, either of the Gaussian type

$$
\begin{aligned}
& \int \prod_{i} d \Gamma^{i} \cdot \exp \left\{\frac{i}{2} \mathfrak{I}_{1 ; i j}[\phi] \Gamma^{i} \cdot \Gamma^{j} \cdot\right\} \Gamma^{k_{1}} \ldots \Gamma^{k_{2 n}} \\
& =\left[\operatorname{det}\left(\mathfrak{I}_{1 ; i j}\right)\right]^{-12} \sum\left(i G^{k j_{1} k j_{2}}\right) \ldots\left(i G^{k j_{2 n-1} k j_{2 n}}\right)
\end{aligned}
$$

where the sum is over all possible pairings of the $\Gamma^{i}, S$, and $G^{i j}[\phi]$ is the full propagator (containing all possible tree insertions), which is the inverse of $\mathfrak{I}_{1 ; i j}[\phi]$ i.e.

$$
\int d x^{\prime \prime} \mathfrak{T}_{1 ; i j}\left[x, x^{\prime \prime} ; \phi\right] G^{j k}\left[x^{\prime \prime}, x^{\prime} ; \phi\right]=\delta_{i}^{k} \delta\left(x-x^{\prime}\right)
$$

2 The results of this section were first circulated in a preprint form in reference [4] 
or of the type

$$
\begin{aligned}
& \int \prod d \omega_{A} \prod d \psi^{A} \exp i\{\bar{\omega} \cdot \mathfrak{A} \cdot \psi+\bar{\omega} \cdot \eta+\bar{\eta} \cdot \psi\} \ldots \psi^{A} \bar{\omega}_{B} \ldots \\
& =\left[\operatorname{det}(i \mathfrak{A})_{B}^{A}\right] \frac{\vec{\delta}}{i \delta \bar{\eta}_{A}} \ldots \exp \{-(i \bar{\eta}) \cdot B \cdot(i \eta)\} \ldots \frac{\overleftarrow{\delta}}{i \delta \eta^{B}}
\end{aligned}
$$

where $B$ is the operator inverse to $(i \mathfrak{U})$ satisfying

$$
\int d x^{\prime \prime}(i \mathfrak{U})_{C}^{A}\left[x, x^{\prime \prime} ; \phi\right] B_{B}^{C}\left[x^{\prime \prime}, x^{\prime} ; \phi\right]=\delta_{B}^{A} \delta\left(x-x^{\prime}\right)
$$

Thus in order to calculate the functional $\amalg[J, \eta]$ we need to know the vertices $\mathfrak{I}_{1 ;\{n\}}(n=3,4, \ldots)$, and $\mathfrak{U}_{B .\{n\}}^{A}(n=1,2, \ldots)$. The former are given in [3]. We turn our attention, therefore, to the vertices $\mathfrak{U}_{B .\{n\}}^{A}$ : Starting from

$$
\mathfrak{U}_{B}^{A}[\phi]=i(\not D)_{B}^{A}[\phi]-m \delta_{B}^{A}+\left(2 F_{\pi}\right)^{-1} \gamma_{5} \gamma_{\mu}\left(\tau_{a}\right)_{B}^{A} s_{a l}(\phi) \partial^{\mu} \phi^{l}
$$

with

$$
(\not D)_{B}^{A}[\phi]=\delta_{B}^{A} \partial^{(B)}-(1 / 8) C_{a b l}(\phi) \partial \phi^{l}\left(\left[\tau_{a}, \tau_{b}\right]\right)_{B}^{A}
$$

we obtain

$$
\begin{aligned}
\mathfrak{A}_{B . k}^{A}[\phi]= & (i / 8)\left(\left[\Gamma^{m}, \Gamma^{n}\right]\right)_{B}^{A} R_{m n k l} \not \phi^{l} \\
& +\left(2 F_{\pi}\right)^{-1} \gamma_{5}\left(\tau_{a}\right)_{B}^{A} s_{a l}(\not)_{k}^{l}
\end{aligned}
$$

where $(\not)_{j}^{i}$ is a differential operator given by

$$
(\not)_{j}^{i}=\left(\delta_{j}^{i} \partial^{(i)}+\Gamma_{j m}^{i} \partial \phi^{m}\right) \delta\left(x_{i}-x_{j}\right)
$$

with $\partial^{(i)}$ indicating differentiation with respect to $x_{i}$, and $R_{m n k l}$ is the Riemann curvature tensor associated with $S^{3}$. Hence, due to the constant curvature situation,

$$
R_{m n k l}=F_{\pi}^{-2}\left(g_{m k} g_{n l}-g_{m l} g_{n k}\right) \delta\left(x_{m}-x_{n}\right) \delta\left(x_{m}-x_{k}\right) \delta\left(x_{m}-x_{l}\right) .
$$

These results can be generalised to the $n^{\text {th }}$ derivative case giving rise to

$$
\begin{aligned}
\mathfrak{U}_{B,\{2 n\}}^{A}[\phi]= & -(1 / 4) \varepsilon_{a b c}\left(\tau_{c}\right)_{B}^{A} s_{a}^{m} S_{b}^{n} R_{m n k_{1} r_{1} \ldots} R_{k_{2 n-2} r_{2 n-1} r_{n}}^{r_{n-1}}(\not)_{k_{2 n}}^{r_{n}} \\
& +\left(1 / 2 F_{\pi}\right) \gamma_{s}\left(\tau_{a}\right)_{B}^{A} S_{a i} R_{k_{1} k_{2} r_{1} \ldots}^{i} \ldots R_{k_{2}-1 k_{2 n} r_{n}}^{r_{n-1}} \partial \phi^{r_{n}}(n \geqq 1)
\end{aligned}
$$

and

$$
\begin{aligned}
\mathfrak{U}_{B .\{2 n+1\}}^{A}[\phi]= & -(1 / 4) \varepsilon_{a b c}\left(\tau_{c}\right)_{B}^{A} s_{a}^{m} s_{b}^{n} R_{m n k_{1} r_{1} \ldots} R_{k_{2 n} k_{2 n-1} r_{n-1}}^{r_{n}} \partial \phi^{r_{n-1}} \\
& +\left(1 / 2 F_{\pi}\right) \gamma_{5}\left(\tau_{a}\right)_{B}^{A} S_{a i} R_{k_{1} k_{2} r_{1}}^{i} \ldots R_{k_{2 n-1} k_{2 n} r_{n}}^{r_{n-1}}\left(\eta_{k_{2 n+1}}^{r_{n}}(n \geqq 1) .\right.
\end{aligned}
$$

In deriving these formulae we have used the fact that the covariant derivative of the Riemann tensor is zero, as well as the fact that the Weyl derivative of the dreibein field is zero, that is

$$
s_{a . k}^{i} \equiv s_{a ; k}^{i}-C_{a b k} s_{b}^{i}=0
$$

Furthermore owing to the form of the generating functional only the symmetric part of the vertex functions makes a contribution. Therefore, complete symmetrization of the chiral indices is understood in (15), and (16).

Acknowledgements. I would like to thank Prof. F. T. Hadjioannou for encouragement and Prof. A. Zachariou for valuable comments. 


\section{References}

1. Meetz, K.: J. Math. Phys. 10, 589 (1969)

2. Honerkamp, J.: Nucl. Phys. B36, 130 (1972)

3. Ecker, G., Honerkamp, J.: Nucl. Phys. B52, 211 (1973)

4. Daniel, M., Horgan, R.R.: Desy 74/2 Preprint

5. Steenrod, N.: The topology of fibre bundles. Princeton: Univ. Press 1951

6. Milnor, J.W., Stasheff, J. D.: Characteristic Classes, Annals of Mathematics Studies. Princeton: Univ. Press 1974

7. Lichnerowicz,A.: Topics on Space-Time, Battelle Recontres: 1967 Lectures in Mathematics and Physics. New York: Benjamin 1968

8. Milnor, J. W.: L’Enseignment Math. 9, 198 (1963)

\section{Communicated by R. Haag}

Received March 1, 1976 